\title{
On the Hyers-Ulam Stability of the First-Order Difference Equation
}

\author{
Soon-Mo Jung and Young Woo Nam \\ Mathematics Section, College of Science and Technology, Hongik University, Sejong 30016, Republic of Korea \\ Correspondence should be addressed to Soon-Mo Jung; smjung@hongik.ac.kr
}

Received 25 May 2016; Accepted 10 July 2016

Academic Editor: Jaeyoung Chung

Copyright (C) 2016 S.-M. Jung and Y. W. Nam. This is an open access article distributed under the Creative Commons Attribution License, which permits unrestricted use, distribution, and reproduction in any medium, provided the original work is properly cited.

We prove Hyers-Ulam stability of the first-order difference equation of the form $x_{i+1}=F\left(i, x_{i}\right)$, where $F$ is a given function with some moderate features. Moreover, we introduce some conditions for the function $F$ under which the difference equation is not stable in the sense of Hyers and Ulam.

\section{Introduction}

The difference equation usually describes the development of a certain phenomenon by recursively defining a sequence, each of whose terms is defined as a function of the preceding terms, once one or more initial terms are known. The difference equation often refers to a specific type of recurrence relation (see [1]).

In 1940, Ulam [2] raised an important problem concerning the stability of group homomorphisms (ref. [3, 4]): given a metric group $(G, \cdot, d)$, a positive number $\varepsilon$, and a function $f: G \rightarrow G$ which satisfies inequality $d(f(x y), f(x) f(y)) \leq \varepsilon$ for all $x, y \in G$, do there exist a homomorphism $a: G \rightarrow$ $G$ and a constant $\delta$ depending only on $G$ and $\varepsilon$ such that $d(a(x), f(x)) \leq \delta$ for all $x \in G$ ?

If the answer to this question is affirmative, the functional equation $a(x y)=a(x) a(y)$ is said to be stable. A first answer to this question was given by Hyers [5] in 1941 who proved that the Cauchy additive equation is stable in Banach spaces. In general, a functional equation is said to be stable in the sense of Hyers and Ulam (or the equation has the HyersUlam stability) if for each solution to the perturbed equation, there exists a solution to the equation that differs from the solution to the perturbed equation with a small error. We refer the reader to [2, 5-11] for the exact definition of Hyers-Ulam stability.

Throughout this paper, we denote by $\mathbb{N}, \mathbb{N}_{0}, \mathbb{R}$, and $\mathbb{C}$ the set of all positive integers, of all nonnegative integers, and of all real numbers and the set of all complex numbers, respectively.

In this paper, we prove Hyers-Ulam stability of the firstorder difference equation of the form

$$
x_{i+1}=F\left(i, x_{i}\right)
$$

for all integers $i \in \mathbb{N}_{0}$, where $F: \mathbb{N}_{0} \times \mathbb{C} \rightarrow \mathbb{C}$ is a given function with some moderate features. More precisely, we prove that if a complex-valued sequence $\left\{a_{i}\right\}_{i \in \mathbb{N}_{0}}$ satisfies inequality

$$
\left|a_{i+1}-F\left(i, a_{i}\right)\right| \leq \varepsilon
$$

for all $i \in \mathbb{N}_{0}$, then there exist a solution $\left\{b_{i}\right\}_{i \in \mathbb{N}_{0}}$ to the difference equation (1) and a positive constant $\delta$ depending only on $F$ and $\varepsilon$ such that

$$
\left|b_{i}-a_{i}\right| \leq \delta
$$

for all $i \in \mathbb{N}_{0}$. As we know, the stability of the difference equation (1) depends on properties of the map $F$. We show in the last part of this paper which condition of $F$ excludes the Hyers-Ulam stability. 


\section{Conditions for Hyers-Ulam Stability}

The principle of recursive definition states that, for any function $g: \mathbb{C} \rightarrow \mathbb{C}$ and any $a_{0} \in \mathbb{C}$, there exists a unique function $f: \mathbb{N}_{0} \rightarrow \mathbb{C}$ such that

$$
\begin{gathered}
f(0)=a_{0}, \\
f(i+1)=g(f(i))
\end{gathered}
$$

for all $i \in \mathbb{N}[12$, Theorem A.5.6]. This principle assures us of the existence and the uniqueness of the sequence $\left\{b_{i}\right\}_{i \in \mathbb{N}_{0}}$ mentioned in Theorem 1.

First, we prove a general theorem that provides us with a powerful tool for proving the Hyers-Ulam stability of a large class of the first-order difference equations.

Theorem 1. Given $\varepsilon>0$, let $F: \mathbb{N}_{0} \times \mathbb{C} \rightarrow \mathbb{C}$ be a function satisfying the condition

$$
|F(i, u)-F(i, v)| \leq \varphi(|u-v|)
$$

for all $i \in \mathbb{N}_{0}$ and all $u, v \in \mathbb{C}$, where $\varphi:[0, \infty) \rightarrow[0, \infty)$ is a monotone increasing function. If a complex-valued sequence $\left\{a_{i}\right\}_{i \in \mathbb{N}_{0}}$ satisfies inequality

$$
\left|a_{i+1}-F\left(i, a_{i}\right)\right| \leq \varepsilon
$$

for all $i \in \mathbb{N}_{0}$, then there exists a complex-valued sequence $\left\{b_{i}\right\}_{i \in \mathbb{N}_{0}}$ satisfying

$$
\begin{gathered}
b_{i+1}=F\left(i, b_{i}\right), \\
\left|b_{i}-a_{i}\right| \leq \psi^{i}\left(\left|b_{0}-a_{0}\right|\right)
\end{gathered}
$$

for all $i \in \mathbb{N}_{0}$, where the function $\psi:[0, \infty) \rightarrow[0, \infty)$ is defined by $\psi(x):=\varphi(x)+\varepsilon$ for all $x \geq 0$ and $\psi^{i}(x)$ denotes the value of the ith iterate of $\psi$ at $x$; that is,

$$
\psi^{i}(x)=(\underbrace{\psi \circ \cdots \circ \psi}_{i})(x) .
$$

Proof. In view of principle of recursive definition, the complex-valued sequence $\left\{b_{i}\right\}_{i \in \mathbb{N}_{0}}$ is uniquely determined via formula (7) provided $b_{0}$ is given.

We will apply an induction on $i$ to prove inequality (8). For $i=1$, it follows from (5), (6), and (7) that

$$
\begin{aligned}
\left|b_{1}-a_{1}\right| & \leq\left|F\left(0, b_{0}\right)-F\left(0, a_{0}\right)\right|+\left|F\left(0, a_{0}\right)-a_{1}\right| \\
& \leq \varphi\left(\left|b_{0}-a_{0}\right|\right)+\varepsilon=\psi\left(\left|b_{0}-a_{0}\right|\right),
\end{aligned}
$$

which we also obtain by putting $i=1$ in (8). We now assume that inequality (8) is true for some $i \in \mathbb{N}$. Then, it follows from (5), (6), (7), and (8) that

$$
\begin{aligned}
\left|b_{i+1}-a_{i+1}\right| & \leq\left|F\left(i, b_{i}\right)-F\left(i, a_{i}\right)\right|+\left|F\left(i, a_{i}\right)-a_{i+1}\right| \\
& \leq \varphi\left(\left|b_{i}-a_{i}\right|\right)+\varepsilon=\psi\left(\left|b_{i}-a_{i}\right|\right) \\
& \leq \psi\left(\psi^{i}\left(\left|b_{0}-a_{0}\right|\right)\right)=\psi^{i+1}\left(\left|b_{0}-a_{0}\right|\right)
\end{aligned}
$$

which proves the validity of (8) for all $i \in \mathbb{N}_{0}$.
Using Theorem 1, we can prove the Hyers-Ulam stability of a class of the first-order difference equations under the condition, $a_{0}=b_{0}$ (see the statement of Corollary 2 for $a_{0}$ and $b_{0}$ ).

Corollary 2. Let $\varphi:[0, \infty) \rightarrow[0, \infty)$ be a monotone increasing mapping such that $\varphi(0)=0$ and

$$
|\varphi(x)-\varphi(y)| \leq K|x-y|
$$

for all $x, y \geq 0$, where $K$ is a positive real constant less than 1. Given $\varepsilon>0$, let $F: \mathbb{N}_{0} \times \mathbb{C} \rightarrow \mathbb{C}$ be a function satisfying condition (5) for all $i \in \mathbb{N}_{0}$ and $u, v \in \mathbb{C}$ and let $\psi:[0, \infty) \rightarrow$ $[0, \infty)$ be defined by $\psi(x):=\varphi(x)+\varepsilon$ for all $x \geq 0$. If a comple $x-$ valued sequence $\left\{a_{i}\right\}_{i \in \mathbb{N}_{0}}$ satisfies inequality (6) for all $i \in \mathbb{N}_{0}$, then there exists a complex-valued sequence $\left\{b_{i}\right\}_{i \in \mathbb{N}_{0}}$ satisfying (7) and

$$
\left|b_{i}-a_{i}\right| \leq \frac{\varepsilon}{1-K}+\frac{K^{i}}{1-K}\left|\psi\left(\left|b_{0}-a_{0}\right|\right)-\right| b_{0}-a_{0}||
$$

for all $i \in \mathbb{N}_{0}$.

Proof. According to Theorem 1, there exists a complex-valued sequence $\left\{b_{i}\right\}_{i \in \mathbb{N}_{0}}$ satisfying (7) and

$$
\left|b_{i}-a_{i}\right| \leq \psi^{i}\left(\left|b_{0}-a_{0}\right|\right)
$$

for all $i \in \mathbb{N}_{0}$.

It follows from (12) that $\psi$ is also a contraction mapping with the Lipschitz constant $K$. By the contraction mapping theorem, we have

$$
\left|\psi^{i}\left(\left|b_{0}-a_{0}\right|\right)-x^{*}\right| \leq \frac{K^{i}}{1-K}\left|\psi\left(\left|b_{0}-a_{0}\right|\right)-\right| b_{0}-a_{0}||
$$

for all $i \in \mathbb{N}_{0}$, where $x^{*}$ is the unique fixed point of $\psi$, from which it follows that

$$
\psi^{i}\left(\left|b_{0}-a_{0}\right|\right) \leq x^{*}+\frac{K^{i}}{1-K}\left|\psi\left(\left|b_{0}-a_{0}\right|\right)-\right| b_{0}-a_{0}||
$$

for each $i \in \mathbb{N}_{0}$. In view of (14) and the last inequality, we obtain

$$
\left|b_{i}-a_{i}\right| \leq x^{*}+\frac{K^{i}}{1-K}\left|\psi\left(\left|b_{0}-a_{0}\right|\right)-\right| b_{0}-a_{0}||
$$

for all $i \in \mathbb{N}_{0}$.

We now assert that $\psi(x) \leq K x+\varepsilon$ for all $x \geq 0$. To prove this assertion, we assume for contradiction that there was $x_{0} \geq 0$ such that $\psi\left(x_{0}\right)>K x_{0}+\varepsilon$. Then we would have

$$
\left|\varphi\left(x_{0}\right)-\varphi(0)\right|=\left|\psi\left(x_{0}\right)-\psi(0)\right|>K\left|x_{0}-0\right|,
$$

which is the contradiction to (12). Hence, we obtain the following inequality for the fixed point $x^{*}$ of $\psi$ :

$$
\begin{aligned}
x^{*} & =\psi\left(x^{*}\right) \leq K x^{*}+\varepsilon \\
\text { or } x^{*} & \leq \frac{\varepsilon}{1-K} .
\end{aligned}
$$

Finally, it follows from (17) and (19) that

$$
\left|b_{i}-a_{i}\right| \leq \frac{\varepsilon}{1-K}+\frac{K^{i}}{1-K}\left|\psi\left(\left|b_{0}-a_{0}\right|\right)-\right| b_{0}-a_{0}||
$$

for all $i \in \mathbb{N}_{0}$. 
Using Corollary 2, we prove the Hyers-Ulam stability of the first-order difference equation (1) under a more explicit condition for $F$ and an additional condition, $a_{0}=b_{0}$ (see the statement of Corollary 3 for $a_{0}$ and $b_{0}$ ).

Corollary 3. Given positive real constants $\varepsilon$ and $K$ with $0<$ $K<1$, let $F: \mathbb{N}_{0} \times \mathbb{C} \rightarrow \mathbb{C}$ be a function satisfying the condition

$$
|F(i, u)-F(i, v)| \leq K|u-v|
$$

for all $i \in \mathbb{N}_{0}$ and $u, v \in \mathbb{C}$. If a complex-valued sequence $\left\{a_{i}\right\}_{i \in \mathbb{N}_{0}}$ satisfies inequality (6) for all $i \in \mathbb{N}_{0}$, then there exists a complex-valued sequence $\left\{b_{i}\right\}_{i \in \mathbb{N}_{0}}$ satisfying (7) and

$$
\left|b_{i}-a_{i}\right| \leq \frac{\varepsilon}{1-K}\left(1+K^{i}\right)+K^{i}\left|b_{0}-a_{0}\right|
$$

for all $i \in \mathbb{N}_{0}$.

Proof. If we define monotone increasing contraction mappings $\varphi, \psi:[0, \infty) \rightarrow[0, \infty)$ by $\varphi(x):=K x$ and $\psi(x):=$ $\varphi(x)+\varepsilon=K x+\varepsilon$, then, by Corollary 2 , we have

$$
\begin{aligned}
\left|b_{i}-a_{i}\right| & \leq \frac{\varepsilon}{1-K}+\frac{K^{i}}{1-K}\left|\psi\left(\left|b_{0}-a_{0}\right|\right)-\right| b_{0}-a_{0}|| \\
& =\frac{\varepsilon}{1-K}+\frac{K^{i}}{1-K} \varepsilon+K^{i}\left|b_{0}-a_{0}\right| \\
& =\frac{\varepsilon}{1-K}\left(1+K^{i}\right)+K^{i}\left|b_{0}-a_{0}\right|
\end{aligned}
$$

for all $i \in \mathbb{N}_{0}$.

Remark 4. If we set $\varepsilon_{n}=\varepsilon, \lambda_{n}=K, L=K$, and $a_{n}(\cdot)=F(n, \cdot)$ in [13, Theorem 2], then we get $K<q<1, n(q)=2$, and $\left|b_{i}-a_{i}\right| \leq M \varepsilon$, where $M \geq K q+1 /(1-q)$, while we get $\left|b_{i}-a_{i}\right| \leq(2 /(1-K)) \varepsilon$ from Corollary 3 provided the initial condition $a_{0}=b_{0}$ is assumed. In general, the result of [13, Theorem 2] is better than that of Corollary 3 .

Example 5. Given positive real constants $\varepsilon$ and $M$ with $0<$ $M<e$, let $F: \mathbb{N}_{0} \times \mathbb{C} \rightarrow \mathbb{C}$ be a function defined by

$$
F(i, u):=\frac{M}{i+1} u e^{-u}
$$

for all $i \in \mathbb{N}_{0}$ and $u \in \mathbb{C}$. Then we have

$$
\begin{aligned}
& |F(i, u)-F(i, v)|=\frac{M}{i+1}\left|u e^{-u}-v e^{-v}\right| \\
& \quad \leq \frac{M}{i+1}\left(\left.\sup _{z \in \mathbb{C}} \frac{d}{d w}\left(w e^{-w}\right)\right|_{w=z}\right)|u-v| \\
& \quad=\frac{M}{i+1} \frac{1}{e}|u-v| \leq \frac{M}{e}|u-v|=K|u-v|
\end{aligned}
$$

for all $i \in \mathbb{N}_{0}$ and $u, v \in \mathbb{C}$, where we set $K=M / e$. Then we have $0<K<1$, and hence inequality (21) holds. According to Corollary 3 , if a complex-valued sequence $\left\{a_{i}\right\}_{i \in \mathbb{N}_{0}}$ satisfies inequality (6) for all $i \in \mathbb{N}_{0}$, then there exists a complexvalued sequence $\left\{b_{i}\right\}_{i \in \mathbb{N}_{0}}$ satisfying (7) and

$$
\begin{aligned}
\left|b_{i}-a_{i}\right| & \leq \frac{\varepsilon}{1-K}\left(1+K^{i}\right)+K^{i}\left|b_{0}-a_{0}\right| \\
& =\frac{e \varepsilon}{e-M}\left(1+\left(\frac{M}{e}\right)^{i}\right)+\left(\frac{M}{e}\right)^{i}\left|b_{0}-a_{0}\right|
\end{aligned}
$$

for all $i \in \mathbb{N}_{0}$.

\section{Conditions for Nonstability}

In this section, we introduce some conditions for the function $F$, under which the first-order difference equation (1) is not stable in the sense of Hyers and Ulam.

We replace inequality (5) in Theorem 1 with another one and prove the counterpart of Theorem 1 in the following theorem.

Theorem 6. Given $\varepsilon>0$, let $F: \mathbb{N}_{0} \times \mathbb{C} \rightarrow \mathbb{C}$ be a function satisfying the condition

$$
|F(i, u)-F(i, v)| \geq \xi(|u-v|)
$$

for all $i \in \mathbb{N}_{0}$ and $u, v \in \mathbb{C}$, where $\xi:[0, \infty) \rightarrow[0, \infty)$ is a monotone increasing function, and let $\zeta:[0, \infty) \rightarrow \mathbb{R}$ be a monotone increasing function defined by

$$
\zeta(x):=\xi(x)-\varepsilon
$$

for all $x \geq 0$. If a complex-valued sequence $\left\{a_{i}\right\}_{i \in \mathbb{N}_{0}}$ satisfies inequality

$$
\left|a_{i+1}-F\left(i, a_{i}\right)\right| \leq \varepsilon
$$

for all $i \in \mathbb{N}_{0}$ and $\zeta\left(\left|b_{1}-a_{1}\right|\right) \geq 0$, then there exists a complexvalued sequence $\left\{b_{i}\right\}_{i \in \mathbb{N}_{0}}$ satisfying

$$
\begin{gathered}
b_{i+1}=F\left(i, b_{i}\right), \\
\left|b_{i+1}-a_{i+1}\right| \geq \zeta^{i}\left(\left|b_{1}-a_{1}\right|\right)
\end{gathered}
$$

for all $i \in \mathbb{N}_{0}$.

Proof. We apply the induction on $i$ to prove inequality (31). Trivially, (31) is true for $i=0$. For $i=1$, we use (27), (29), and (30) to show that

$$
\begin{aligned}
\left|b_{2}-a_{2}\right| & \geq\left|F\left(1, b_{1}\right)-F\left(1, a_{1}\right)\right|-\left|a_{2}-F\left(1, a_{1}\right)\right| \\
& \geq \xi\left(\left|b_{1}-a_{1}\right|\right)-\varepsilon=\zeta\left(\left|b_{1}-a_{1}\right|\right) ;
\end{aligned}
$$

that is, inequality (31) holds for $i=1$. We now assume that inequality

$$
\left|b_{k+1}-a_{k+1}\right| \geq \zeta^{k}\left(\left|b_{1}-a_{1}\right|\right)
$$

is true for all $k \in\{0,1, \ldots, i-1\}$, where $i$ is a positive integer. Then, it follows from (27), (29), and (30) that

$$
\begin{aligned}
\left|b_{i+1}-a_{i+1}\right| & \geq\left|F\left(i, b_{i}\right)-F\left(i, a_{i}\right)\right|-\left|a_{i+1}-F\left(i, a_{i}\right)\right| \\
& \geq \xi\left(\left|b_{i}-a_{\mathrm{i}}\right|\right)-\varepsilon=\zeta\left(\left|b_{i}-a_{i}\right|\right) \\
& \geq \zeta\left(\zeta^{i-1}\left(\left|b_{1}-a_{1}\right|\right)\right)=\zeta^{i}\left(\left|b_{1}-a_{1}\right|\right),
\end{aligned}
$$

which proves the validity of (31) for all $i \in \mathbb{N}_{0}$. 
Lemma 7. Let $\zeta:[C, \infty) \rightarrow[C, \infty)$ be an increasing continuous function for some $C \in \mathbb{R}$. Assume that $\zeta(C)>C$ and $\zeta$ has fixed points which are greater than $C$; that is, $P=$ $\{x>C: \zeta(x)=x\}$ is nonempty. If $x_{*}:=\inf P$, then $\zeta^{i}(x) \leq x_{*}$ for all $x \in\left[C, x_{*}\right]$ and for all $i \in \mathbb{N}_{0}$. Moreover,

$$
\lim _{i \rightarrow \infty} \zeta^{i}(x)=x_{*}
$$

Proof. The fact that $\zeta$ is increasing and continuous implies that $\zeta(x) \leq \zeta\left(x_{*}\right)=x_{*}$ for all $x \in\left[c_{0}, x_{*}\right]$, where $c_{0} \in\left[C, x_{*}\right]$. Denoting $c_{i}=\zeta^{i}\left(c_{0}\right)$ for each $i \in \mathbb{N}_{0}$, assume that there exists $m \in \mathbb{N}_{0}$ satisfying

$$
c_{m} \leq x_{*}<c_{m+1}
$$

for some $c_{0} \in\left[C, x_{*}\right]$. Since $\zeta$ is an increasing continuous function, we have the following inequality:

$$
x_{*}<c_{m+1}=\zeta\left(c_{m}\right) \leq \zeta\left(x_{*}\right)=x_{*},
$$

which is the contradiction. Consequently, $x_{*}$ is an upper bound of the sequence $\left\{\zeta^{i}(x)\right\}_{i \in \mathbb{N}_{0}}$ for each $x \in\left[C, x_{*}\right]$.

Next, we claim that $\left\{\zeta^{i}(x)\right\}_{i \in \mathbb{N}_{0}}$ is an increasing sequence for each $x \in\left[C, x_{*}\right]$. Suppose for contradiction that there exists $m \in \mathbb{N}_{0}$ such that $c_{m}>\zeta\left(c_{m}\right)=c_{m+1}$ for some $c_{0} \in$ $\left[C, x_{*}\right]$. If we define $g(x):=\zeta(x)-x$, then the following are satisfied:

(i) $g(x)$ is continuous.

(ii) $g(C)=\zeta(C)-C>0$.

(iii) $g\left(c_{m}\right)=\zeta\left(c_{m}\right)-c_{m}=c_{m+1}-c_{m}<0$.

Thus, by the intermediate value theorem for $g$, the function $\zeta$ has a fixed point in $\left(C, c_{m}\right)$ and $c_{m} \leq x_{*}$. It is contrary to the assumption that $x_{*}$ is the infimum of $P$. Therefore, we conclude that $\left\{\zeta^{i}(x)\right\}_{i \in \mathbb{N}_{0}}$ is an increasing sequence for each $x \in\left[C, x_{*}\right]$.

Since the sequence $\left\{\zeta^{i}(x)\right\}_{i \in \mathbb{N}_{0}}$ is an increasing sequence

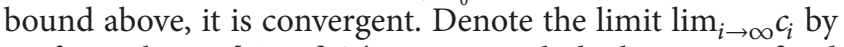
$c_{\infty}$ for each $c_{0} \in\left[C, x_{*}\right]$. Then, we conclude that $c_{\infty}$ is a fixed point of $\zeta$ by considering the relation

$$
c_{\infty}=\lim _{i \rightarrow \infty} c_{i}=\lim _{i \rightarrow \infty} c_{i+1}=\lim _{i \rightarrow \infty} \zeta\left(c_{i}\right)=\zeta\left(c_{\infty}\right) .
$$

Hence, we obtain $x_{*} \leq c_{\infty}$ by the minimality of $x_{*}$. On the other hand, $x_{*}$ is an upper bound of $c_{m}$; that is, $c_{m} \leq x_{*}$ for all $m \in \mathbb{N}_{0}$. Thus, we get

$$
c_{\infty}=\lim _{i \rightarrow \infty} c_{i} \leq x_{*},
$$

which implies that $\lim _{i \rightarrow \infty} \zeta^{i}\left(c_{0}\right)=c_{\infty}=x_{*}$ for each $c_{0} \epsilon$ $\left[C, x_{*}\right]$.

Lemma 8. Let $L$, $\beta$, and $\varepsilon$ be constants with $L>0,0<\beta<1$, and $0<\varepsilon<(L / 2)^{1 /(1-\beta)}$. Then, the function $\zeta:[0, \infty) \rightarrow \mathbb{R}$, defined by $\zeta(x)=L x^{\beta}-\varepsilon$, has two distinct fixed points.
Proof. Since $\varepsilon<(L / 2)^{1 /(1-\beta)}$, we have $\zeta(0)=-\varepsilon<0$ and

$$
\zeta(\varepsilon)=L \varepsilon^{\beta}-\varepsilon=\varepsilon\left(L \varepsilon^{\beta-1}-1\right)>\varepsilon\left(L \frac{2}{L}-1\right)=\varepsilon .
$$

Since $\zeta(\varepsilon)-\varepsilon>0, \zeta$ has a fixed point $x_{1}^{*}$ in $(0, \varepsilon)$ by the intermediate value theorem for the continuous function $\zeta(x)-x$. Moreover, if $x \geq L^{1 /(1-\beta)}$, then we have $x^{1-\beta} \geq L$ and hence, $x \geq L x^{\beta}>L x^{\beta}-\varepsilon=\zeta(x)$; that is, $\zeta(x)-x<0$ for each $x \geq L^{1 /(1-\beta)}$. Hence, $\zeta$ has another fixed point $x_{2}^{*}$ in $\left(\varepsilon, L^{1 /(1-\beta)}\right)$.

Corollary 9. Let $L, \beta$, and $\varepsilon$ be constants with $L>0,0<\beta<$ 1 , and $0<\varepsilon<(L / 3)^{1 /(1-\beta)}$. The function $\zeta(x)=L x^{\beta}-\varepsilon$ has a fixed point $x_{1}^{*}$ in the interval $(0, \varepsilon / 2)$ and another one $x_{2}^{*}$ in $\left((L / 2)^{1 /(1-\beta)}, L^{1 /(1-\beta)}\right)$.

Proof. We see that $\zeta(0)=-\varepsilon<0$ and

$$
\begin{aligned}
\zeta\left(\frac{\varepsilon}{2}\right) & =L \frac{\varepsilon^{\beta}}{2^{\beta}}-\varepsilon>L \frac{\varepsilon^{\beta}}{2}-\varepsilon=\frac{\varepsilon}{2}\left(L \varepsilon^{\beta-1}-2\right) \\
& >\frac{\varepsilon}{2}\left(L \frac{3}{L}-2\right)=\frac{\varepsilon}{2} .
\end{aligned}
$$

Hence, if we apply the intermediate value theorem with the continuous function $\eta(x)=\zeta(x)-x$, then we have $\eta(0)<0$ and $\eta(\varepsilon / 2)>0$, which implies that $\zeta$ has a fixed point in the interval $(0, \varepsilon / 2)$.

Similarly, we have

$$
\zeta\left(L^{1 /(1-\beta)}\right)=L \cdot L^{\beta /(1-\beta)}-\varepsilon=L^{1 /(1-\beta)}-\varepsilon<L^{1 /(1-\beta)}
$$

and moreover,

$$
\begin{aligned}
\zeta\left(\left(\frac{L}{2}\right)^{1 /(1-\beta)}\right) & =2 \cdot \frac{L}{2}\left(\frac{L}{2}\right)^{\beta /(1-\beta)}-\varepsilon \\
& =2\left(\frac{L}{2}\right)^{1 /(1-\beta)}-\varepsilon \\
& >2\left(\frac{L}{2}\right)^{1 /(1-\beta)}-\left(\frac{L}{2}\right)^{1 /(1-\beta)} \\
& =\left(\frac{L}{2}\right)^{1 /(1-\beta)} .
\end{aligned}
$$

We also apply the intermediate value theorem to $\eta$ on the interval $\left[(L / 2)^{1 /(1-\beta)}, L^{1 /(1-\beta)}\right]$. Then $\zeta$ has another fixed point $x_{2}^{*}$ in $\left((L / 2)^{1 /(1-\beta)}, L^{1 /(1-\beta)}\right)$.

Remark 10. The fixed point $x_{2}^{*}$ of $\zeta$ in Corollary 9 is contained in the interval $\left((L / 2)^{1 /(1-\beta)}, L^{1 /(1-\beta)}\right)$ independent of $\varepsilon$ which runs over the interval $\left(0,(L / 3)^{1 /(1-\beta)}\right)$. Since $L^{1 /(1-\beta)}$ is a fixed point of the mapping $\xi(x)=L x^{\beta}$ and $\zeta$ has only two fixed points, $x_{2}^{*}$ converges to $L^{1 /(1-\beta)}$ as $\varepsilon \rightarrow 0$.

In the following theorem, we introduce a condition for the function $F$, under which the first-order difference equation (1) is not stable in the sense of Hyers and Ulam. 
Theorem 11. Let $L$ and $\beta$ be constants with $L>0$ and $0<\beta<$ 1. Given $\varepsilon>0$, let $F: \mathbf{N}_{0} \times \mathbf{C} \rightarrow \mathbf{C}$ be a function satisfying the condition

$$
|F(i, u)-F(i, v)| \geq L|u-v|^{\beta}
$$

for all $i \in \mathbf{N}_{0}$ and $u, v \in \mathbf{C}$. If a complex-valued sequence $\left\{a_{i}\right\}_{i \in \mathbf{N}_{0}}$ satisfies inequality

$$
\left|a_{i+1}-F\left(i, a_{i}\right)\right| \leq \varepsilon
$$

for all $i \in \mathbf{N}_{0}$, then for every sequence $\left\{b_{i}\right\}_{i \in \mathbf{N}_{0}}$ satisfying $b_{i+1}=$ $F\left(i, b_{i}\right)$ and $\left|a_{0}-b_{0}\right| \geq \varepsilon / 2$, it holds that

$$
\liminf _{i \rightarrow \infty}\left|b_{i}-a_{i}\right| \geq\left(\frac{L}{3}\right)^{1 /(1-\beta)} .
$$

Proof. Let $\varepsilon$ be a constant with $0<\varepsilon<(L / 3)^{1 /(1-\beta)}$. Denote $\xi$ and $\zeta$ as follows:

$$
\begin{aligned}
& \xi(x)=L x^{\beta}, \\
& \zeta(x)=L x^{\beta}-\varepsilon .
\end{aligned}
$$

We set $\varepsilon_{i}=\left|a_{i+1}-F\left(i, a_{i}\right)\right|$ for all $i \in \mathbb{N}_{0}$ and in view of (45), without loss of generality, we can choose $\varepsilon:=\sup _{i \in \mathbb{N}_{0}} \varepsilon_{i}$. Then there exists $k \in \mathbb{N}_{0}$ such that

$$
\frac{\varepsilon}{2}<\varepsilon_{k} \leq \varepsilon
$$

Thus we may assume that $\varepsilon / 2<\varepsilon_{0} \leq \varepsilon$. By Lemma $8, \zeta$ has two distinct fixed points $x_{1}^{*}$ and $x_{2}^{*}$ with $x_{1}^{*}<x_{2}^{*}$. Since $\zeta$ is an increasing continuous function and $\varepsilon>0$, there is the point $x_{0}$ satisfying $\zeta\left(x_{0}\right)=0$. Moreover, Corollary 9 implies that

$$
0<x_{0}<x_{1}^{*}<\frac{\varepsilon}{2}<\varepsilon<\left(\frac{L}{3}\right)^{1 /(1-\beta)}<x_{2}^{*}<L^{1 /(1-\beta)} .
$$

By the triangular inequality and Theorem 6, we have

$$
\begin{aligned}
\left|b_{1}-a_{1}\right| & \geq\left|F\left(0, b_{0}\right)-F\left(0, a_{0}\right)\right|-\left|a_{1}-F\left(0, a_{0}\right)\right| \\
& \geq \xi\left(\left|b_{0}-a_{0}\right|\right)-\varepsilon_{0} .
\end{aligned}
$$

Inequality (41) implies that

$$
\begin{aligned}
\xi\left(\frac{\varepsilon}{2}\right) & =\zeta\left(\frac{\varepsilon}{2}\right)+\varepsilon>\frac{3}{2} \varepsilon, \\
\xi\left(\frac{\varepsilon}{2}\right)-\varepsilon_{0} & >\frac{3}{2} \varepsilon-\varepsilon_{0} \geq \frac{3}{2} \varepsilon-\varepsilon=\frac{\varepsilon}{2}, \\
\zeta\left(\frac{\varepsilon}{2}\right) & >\frac{\varepsilon}{2}>0 .
\end{aligned}
$$

Since the functions $\xi(x)-\varepsilon_{0}$ and $\zeta(x)$ are increasing functions, we obtain

$$
\begin{array}{r}
\xi\left(\left|b_{0}-a_{0}\right|\right)-\varepsilon_{0}>\frac{\varepsilon}{2}, \\
\zeta\left(\left|b_{0}-a_{0}\right|\right)>0
\end{array}
$$

for all $\left|b_{0}-a_{0}\right| \in[\varepsilon / 2, \infty)$. Then, in view of (49), (50), and (52), $\left|b_{1}-a_{1}\right|$ is greater than the fixed point $x_{1}^{*}$; that is,

$$
\left|b_{1}-a_{1}\right| \geq \xi\left(\left|b_{0}-a_{0}\right|\right)-\varepsilon_{0}>\frac{\varepsilon}{2}>x_{1}^{*} \text {. }
$$

Moreover, since $\zeta$ is increasing, we have

$$
\zeta\left(\left|b_{1}-a_{1}\right|\right) \geq \zeta\left(\frac{\varepsilon}{2}\right)>\frac{\varepsilon}{2}>0 .
$$

By Theorem 6, inequality $\left|b_{i+1}-a_{i+1}\right| \geq \zeta^{i}\left(\left|b_{1}-a_{1}\right|\right)$ holds for all $i \in \mathbb{N}_{0}$ and by putting $C=\varepsilon / 2$ and $x_{*}=x_{2}^{*}$ in Lemma 7 and taking (41) into account, $\zeta^{i}\left(\left|b_{1}-a_{1}\right|\right)$ converges to $x_{2}^{*}$. Hence, by Theorem 6 and (49), we get

$$
\left|b_{i+1}-a_{i+1}\right| \geq \zeta^{i}\left(\left|b_{1}-a_{1}\right|\right) \geq\left(\frac{L}{3}\right)^{1 /(1-\beta)}
$$

for all sufficiently large integers $i$.

Remark 12. Theorem 11 can be regarded as a nonstability result because this theorem shows that the difference $\left|b_{i}-a_{i}\right|$ can be quite large even in the case when $\varepsilon$ is very small. An analogous nonstability result can be deduced from [14, Theorem 1] when $\beta=1$ and $L=1$. However, this is not the case when $\beta=1$ and $L>1$ because [13, Theorem 1] shows that the sequence has Hyers-Ulam stability.

\section{Competing Interests}

The authors declare that they have no competing interests.

\section{Authors' Contributions}

All authors contributed equally to the writing of this paper. All authors read and approved the final paper.

\section{Acknowledgments}

This research was supported by Basic Science Research Program through the National Research Foundation of Korea (NRF) funded by the Ministry of Education (no. 2015R1D1A1A02061826).

\section{References}

[1] S. N. Elaydi, An Introduction to Difference Equations, Springer, New York, NY, USA, 3rd edition, 2005.

[2] S. M. Ulam, A Collection of Mathematical Problems, Interscience Publishers, New York, NY, USA, 1960, Reprinted as: Problems in Modern Mathematics, John Wiley \& Sons, New York, NY, USA, 1964.

[3] D. Popa, "Hyers-Ulam-Rassias stability of a linear recurrence," Journal of Mathematical Analysis and Applications, vol. 309, no. 2, pp. 591-597, 2005.

[4] D. Popa, "Hyers-Ulam stability of the linear recurrence with constant coefficients," Advances in Difference Equations, vol. 2005, Article ID 407076, 2005.

[5] D. H. Hyers, "On the stability of the linear functional equation," Proceedings of the National Academy of Sciences of the United States of America, vol. 27, pp. 222-224, 1941. 
[6] S. Czerwik, Functional Equations and Inequalities in Several Variables, World Scientific, Singapore, 2002.

[7] G. Z. Eskandani, P. Găvruta, J. M. Rassias, and R. Zarghami, "Generalized Hyers-Ulam stability for a general mixed functional equation in quasi- $\beta$-normed spaces," Mediterranean Journal of Mathematics, vol. 8, no. 3, pp. 331-348, 2011.

[8] P. Găvruta, "A generalization of the Hyers-Ulam-Rassias stability of approximately additive mappings," Journal of Mathematical Analysis and Applications, vol. 184, no. 3, pp. 431-436, 1994.

[9] D. H. Hyers, G. Isac, and Th. M. Rassias, Stability of Functional Equations in Several Variables, Birkhäuser, Boston, Mass, USA, 1998.

[10] S.-M. Jung, Hyers-Ulam-Rassias Stability of Functional Equations in Nonlinear Analysis, vol. 48 of Springer Optimization and Its Applications, Springer, New York, NY, USA, 2011.

[11] Th. M. Rassias, "On the stability of the linear mapping in Banach spaces," Proceedings of the American Mathematical Society, vol. 72, no. 2, pp. 297-300, 1978 .

[12] T. B. Singh, Elements of Topology, CRC Press, New York, NY, USA, 2013.

[13] J. Brzdęk, D. Popa, and B. Xu, "The Hyers-Ulam stability of nonlinear recurrences," Journal of Mathematical Analysis and Applications, vol. 335, no. 1, pp. 443-449, 2007.

[14] J. Brzdęk, D. Popa, and B. Xu, "On nonstability of the linear recurrence of order one," Journal of Mathematical Analysis and Applications, vol. 367, no. 1, pp. 146-153, 2010. 


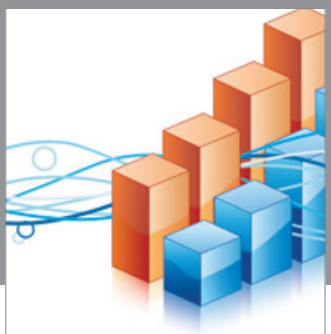

Advances in

Operations Research

vatem alat4

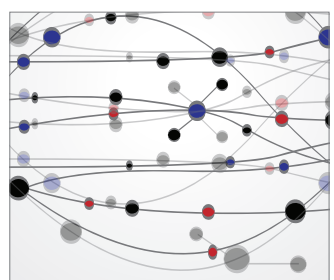

\section{The Scientific} World Journal
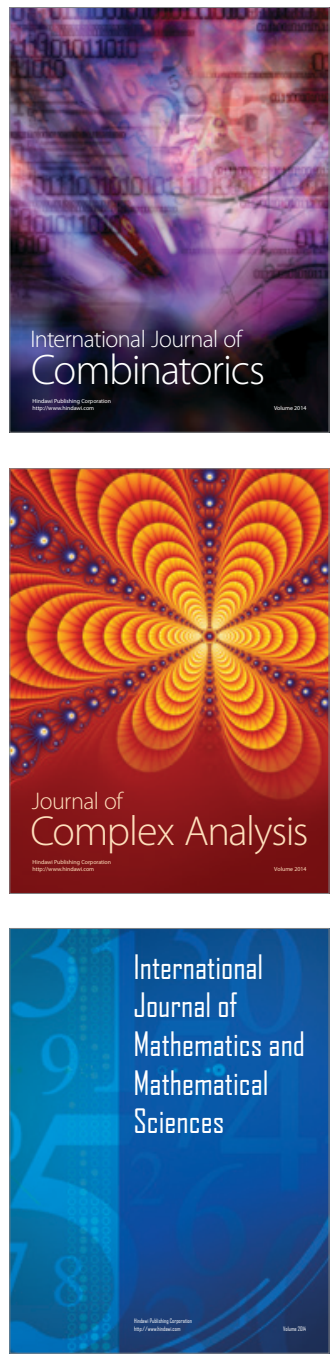
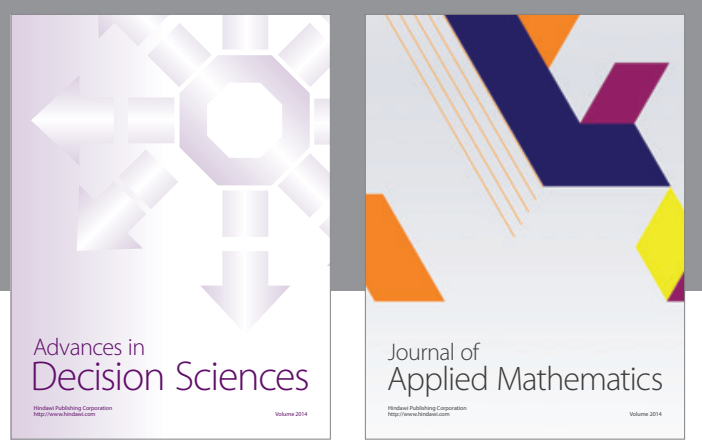

Algebra

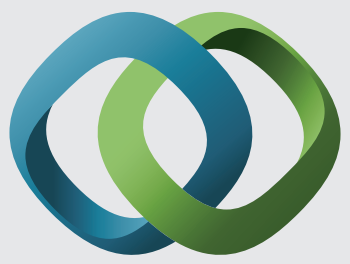

\section{Hindawi}

Submit your manuscripts at

http://www.hindawi.com
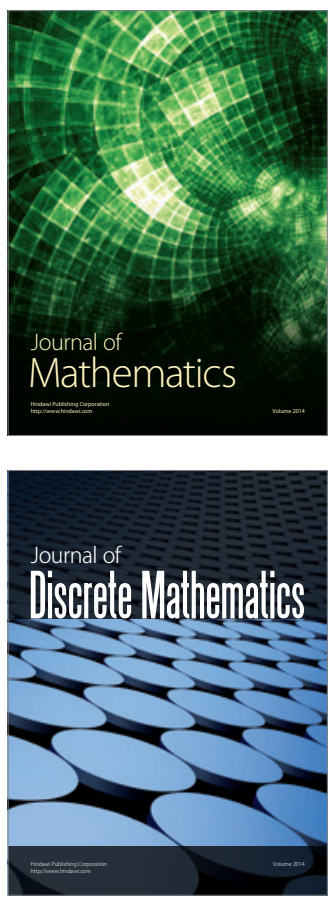

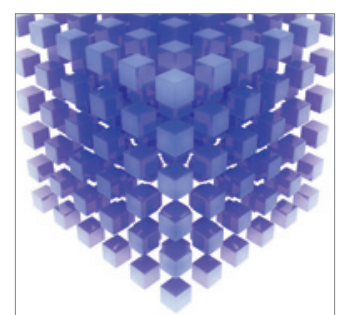

Mathematical Problems in Engineering
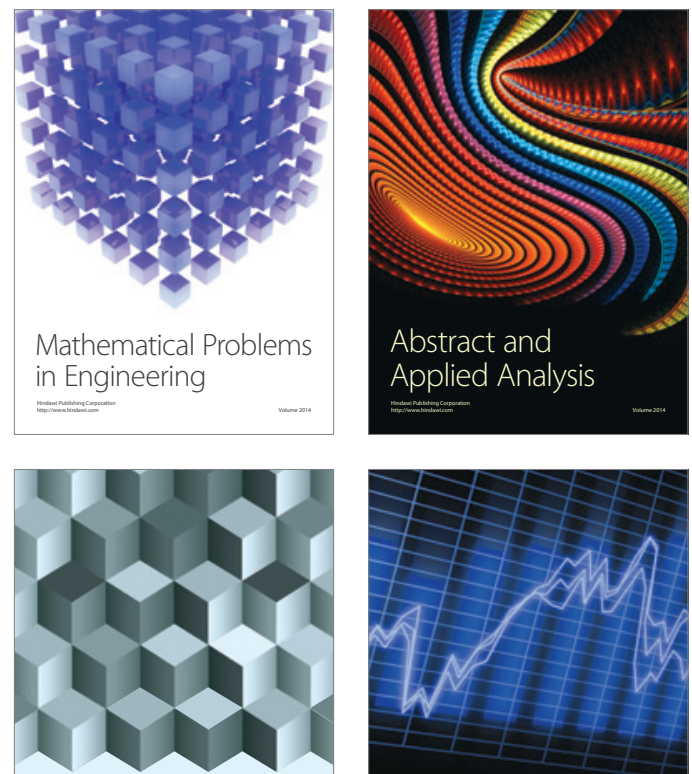

Journal of

Function Spaces

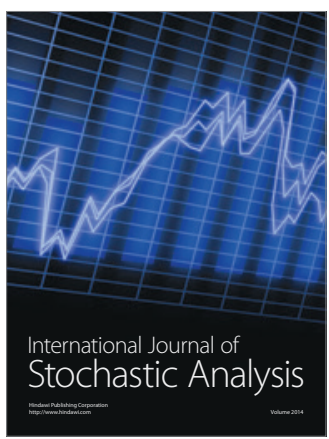

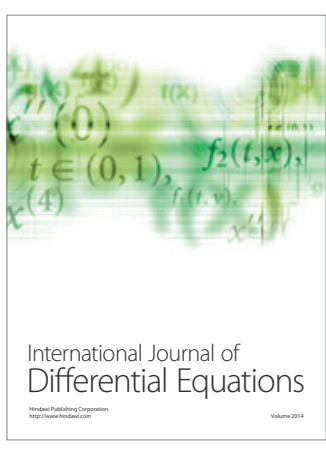
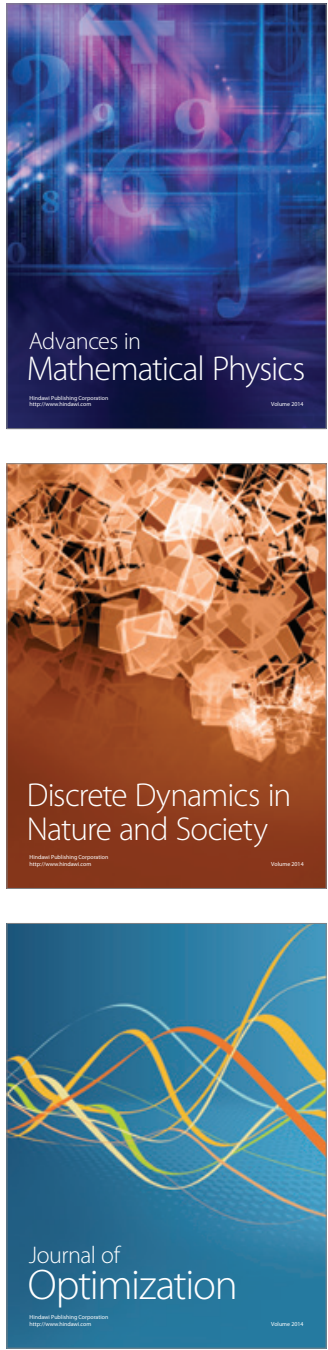